\title{
Serum Amyloid A Protein as a Potential Biomarker in predicting acute onset and association with in- hospital death in acute aortic dissection
}

\section{CURRENT STATUS: ACCEPTED}

BMC Cardiovascular Disorders $\triangle$ BMC series

Yuchen $\mathrm{He}$

Chinese Academy of Medical Sciences and Peking Union Medical College

Changcheng $\mathrm{Ma}$

Shengjing Hospital of China Medical University

Jia Xing

China Medical University

Shiyue Wang

the First Hospital of China Medical University

Ji Chao

Shengjing Hospital of China Medical University

Yanshuo Han

Shengjing Hospital of China Medical University

yanshuohan@cmu.edu.cnCorresponding Author

ORCiD: https://orcid.org/0000-0002-4897-2998

Jian Zhang

the First Hospital of China Medical University

DOI:

$10.21203 / \mathrm{rs} .2 .17288 / \mathrm{v} 1$

SUBJECT AREAS

Cardiac \& Cardiovascular Systems

KEYWORDS

Serum amyloid A, Acute aortic dissection, Biomarker, Inflammation 
Abstract

Background: Acute aortic dissection (AAD) is a life-threatening disorder in vascular surgery with a high early mortality. Serum amyloid A (SAA) is a kind of acute-phase protein with a rapid diagnostic value in other diseases. However, the researches on the performance of SAA for the diagnosis of AAD is still lacking. This retrospective study aimed to evaluate the SAA levels and further explore its potential diagnostic role in AAD patients.

Methods: SAA levels were measured by enzyme-linked immunosorbent assay (ELISA) in 63 controls and 87 AAD patients. Laboratory examinations were also performed. And relative clinical information was collected from participants included in this study.

Results: SAA levels were significantly higher in AAD patients than those in healthy controls. SAA levels were independently associated with the risk of AAD. There was a positive significant correlation between SAA and $C$ reactive protein $(R=0.442$, and $P=0.001)$. Based on receiver-operating characteristic $(R O C)$ analysis, the area under the curve (AUC) of SAA for the diagnosis of AAD were 0.942 with optimal cut-off points of $0.427 \mathrm{mg} / \mathrm{L}$. For in-hospital mortality, the AUC of SAA were 0.732 with optimal cut-off points of $0.500 \mathrm{mg} / \mathrm{L}$. According to logistic regression analysis, higher SAA levels represent a higher risk of in-hospital mortality $(\mathrm{OR}=1.25 ; 95 \% \mathrm{Cl}: 1.07-1.47 ; \mathrm{P}=0.005)$.

Conclusion: Our findings demonstrated that SAA levels were significantly enhanced in AAD. SAA was closely correlated with inflammatory parameters and coagulation-related parameters in AAD.

Furthermore, SAA could be a potential bio-marker for identifying AAD in the early diagnosis. Finally, SAA $>5.0 \mathrm{mg} / \mathrm{L}$ are independently related to AAD in-hospital mortality.

\section{Full Text}

Due to technical limitations, full-text HTML conversion of this manuscript could not be completed. However, the manuscript can be downloaded and accessed as a PDF. Tables 
Table 1 Demographic and laboratory parameters of participants included in this study

\begin{tabular}{|c|c|c|c|c|}
\hline & Control $(n=63)$ & Angina $(n=20)$ & $\operatorname{AAD}(n=87)$ & $\begin{array}{l}\bar{P} . \\
A \\
A\end{array}$ \\
\hline Male & $43(68.25 \%)$ & $15(75.00 \%)$ & $65(74.71 \%)$ & 0. \\
\hline Hypertension & 21 (33.33\%) & $8(40.00 \%)$ & $54(62.69 \%)$ & 0. \\
\hline Smoking & 19 (30.16\%) & $7(35.00 \%)$ & 31 (35.63) & 0. \\
\hline DM & 10 (15.87\%) & $6(30.00 \%)$ & 26 (29.89\%) & 0. \\
\hline Age & $53.44 \pm 11.52$ & $57.80 \pm 2.11$ & $53.87 \pm 11.42$ & 0. \\
\hline BMI & $21.8 \pm 3.68$ & $23.70 \pm 0.73$ & $22.87 \pm 5.14$ & 0 . \\
\hline HR & $78.06 \pm 9.6$ & $79.45 \pm 1.68$ & $89.46 \pm 12.68$ & $<$ \\
\hline WBC & $5.83 \pm 0.77$ & $6.39 \pm 0.40$ & $10.74 \pm 4.24$ & $<$ \\
\hline HGB & $150.98 \pm 17.68$ & $135.50 \pm 4.08$ & $132.39 \pm 20.97$ & $<$ \\
\hline PLT & $173.3 \pm 43.14$ & $201.35 \pm 13.27$ & $190.59 \pm 68.15$ & 0. \\
\hline FPG & $4.97 \pm 4.87$ & $5.54 \pm 0.24$ & $7.21 \pm 2.42$ & 0. \\
\hline SAA & $0.36 \pm 0.08$ & $0.72 \pm 0.40$ & $4.43 \pm 0.31$ & $\begin{array}{l}< \\
0 .\end{array}$ \\
\hline \multicolumn{5}{|c|}{$\begin{array}{l}\text { Note, AAD-acute aortic dissection; TAAD-type A aortic dissection; TBAD-type B aortic dissection; BMI-body mass index; HI } \\
\text { platelet; FPG-fast plasma glucose; SAA-serum amyloid A }\end{array}$} \\
\hline \multicolumn{5}{|c|}{ Table 2 Comparison of SAA levels between AAD and control group, AAD and angina group stratified by cardiovascular ris! } \\
\hline \multirow[t]{2}{*}{ Variables } & & Control & Angina & $\overline{\mathrm{AAL}}$ \\
\hline & & $\mathrm{SAA}(\mathrm{ng} / \mathrm{mg})$ & $\mathrm{SAA}(\mathrm{ng} / \mathrm{mg})$ & $\overline{\mathrm{SA}}$ \\
\hline \multirow[t]{2}{*}{ Age } & $<60 y$ & $0.418 \pm 0.113$ & $0.661 \pm 0.333$ & $\overline{4.4}$ \\
\hline & $\geq 60 y$ & $0.263 \pm 0.084$ & $0.632 \pm 0.323$ & 6.8 \\
\hline \multirow[t]{2}{*}{ Gender } & male & $0.375 \pm 0.093$ & $0.697 \pm 0.295$ & 5.5 \\
\hline & female & $0.332 \pm 0.146$ & $0.446 \pm 0.244$ & 5.2 \\
\hline \multirow[t]{2}{*}{ BMI } & $<25$ & $0.300 \pm 0.067$ & $0.652 \pm 0.301$ & 5.8 \\
\hline & $\geq 25$ & $0.652 \pm 0.316$ & $0.630 \pm 0.364$ & 4.3 \\
\hline \multirow[t]{2}{*}{ Hypertension } & Yes & $0.445 \pm 0.164$ & $0.808 \pm 0.204$ & 4.8 \\
\hline & No & $0.319 \pm 0.085$ & $0.535 \pm 0.342$ & $6.3^{\prime}$ \\
\hline \multirow[t]{2}{*}{ Smoking history } & Yes & $0.510 \pm 0.215$ & $0.728 \pm 0.326$ & 7.6 \\
\hline & No & $0.297 \pm 0.063$ & $0.516 \pm 0.278$ & 4.2 \\
\hline \multirow[t]{2}{*}{ DM } & Yes & $0.232 \pm 0.093$ & $0.566 \pm 0.356$ & 8.1 \\
\hline & No & $0.385 \pm 0.093$ & $0.677 \pm 0.311$ & 4.3 \\
\hline
\end{tabular}

Note, AAD-acute aortic dissection; BMI-body mass index; DM-diabetes mellitus; SAA-serum amyloid A 
Table 3 Comparisons of blood parameters between different sub-groups in AAD patient group

\begin{tabular}{|c|c|c|c|c|c|c|c|}
\hline Parameters & TAAD & TBAD & $P$ value & less than $6 \mathrm{~h}$ & more than $6 \mathrm{~h}$ & $P$ value & $\begin{array}{r}\text { commu } \\
\text { fa } \\
\text { Iun }\end{array}$ \\
\hline WBC, $\times 10^{9} / \mathrm{L}$ & $11.54 \pm 4.5$ & $10.06 \pm 3.92$ & 0.198 & $10.73 \pm 4.75$ & $10.75 \pm 3.78$ & 0.917 & 12.1 \\
\hline BA/WBC, \% & $0.20 \pm 0.23$ & $0.28 \pm 0.34$ & 0.646 & $0.24 \pm 0.3$ & $0.25 \pm 0.30$ & 0.723 & $0.3 \pm$ \\
\hline EO/WBC, \% & $1.37 \pm 1.65$ & $0.89 \pm 1.51$ & 0.139 & $0.95 \pm 1.37$ & $1.26 \pm 1.76$ & 0.409 & $1.04:$ \\
\hline MO/WBC, \% & $13.29 \pm 14.10$ & $8.48 \pm 9.03$ & $0.002 * *$ & $9.29 \pm 7.37$ & $11.96 \pm 14.7$ & 0.909 & $15.02:$ \\
\hline LY/WBC, \% & $14.87 \pm 9.26$ & $15.98 \pm 9.1$ & 0.462 & $14.76 \pm 8.5$ & $16.1 \pm 9.72$ & 0.596 & 14.24 \\
\hline NE/WBC, \% & $69.92 \pm 15.24$ & $74.31 \pm 12.97$ & 0.198 & $74.49 \pm 12.41$ & $70.31 \pm 15.41$ & 0.275 & $69.4 \pm$ \\
\hline $\mathrm{RBC}, \times 10^{12} / \mathrm{L}$ & $4.26 \pm 0.64$ & $4.52 \pm 0.76$ & $0.014 *$ & $4.36 \pm 0.65$ & $4.43 \pm 0.77$ & 0.730 & $4.32:$ \\
\hline HGB, g/L & $128.63 \pm 17.86$ & $135.61 \pm 23.03$ & 0.052 & $134.92 \pm 18.44$ & $130.13 \pm 23$ & 0.328 & 126.73 \\
\hline $\mathrm{MCH}, \mathrm{pg}$ & $30.45 \pm 3.12$ & $30.49 \pm 1.94$ & 0.373 & $31.06 \pm 2.15$ & $29.94 \pm 2.75$ & $0.015^{*}$ & 29.89 \\
\hline $\mathrm{MCV}, \mathrm{fL}$ & $91.66 \pm 8.00$ & $92.11 \pm 5.52$ & 0.696 & $93.2 \pm 5.67$ & $90.74 \pm 7.43$ & 0.181 & $90.10:$ \\
\hline $\mathrm{MCHC}, \mathrm{g} / \mathrm{L}$ & $331.77 \pm 11.11$ & $331.12 \pm 11.83$ & 0.731 & $333.17 \pm 9.78$ & $329.85 \pm 12.65$ & 0.348 & $330.8=$ \\
\hline HCT, L/L & $0.38 \pm 0.06$ & $0.41 \pm 0.07$ & 0.083 & $0.41 \pm 0.06$ & $0.39 \pm 0.08$ & 0.428 & $0.38=$ \\
\hline RDW, \% & $13.73 \pm 2.43$ & $13.25 \pm 1.3$ & 0.864 & $13.38 \pm 1.47$ & $13.51 \pm 2.17$ & 0.753 & 14.26 \\
\hline $\mathrm{PLT}, \times 10^{9} / \mathrm{L}$ & $199.34 \pm 67.05$ & $183.12 \pm 69$ & 0.196 & $177.75 \pm 64.26$ & $202.15 \pm 70.25$ & 0.218 & 166.07 \\
\hline MPV, fL & $9.48 \pm 1.13$ & $9.93 \pm 1.67$ & 0.158 & $9.95 \pm 1.43$ & $9.52 \pm 1.46$ & 0.380 & $9.51:$ \\
\hline $\mathrm{PCT}, \mathrm{L} / \mathrm{L}$ & $1.02 \pm 2.26$ & $0.36 \pm 1.11$ & 0.076 & $0.80 \pm 1.99$ & $0.56 \pm 1.54$ & 0.264 & $1.22:$ \\
\hline PDW, 10GSD & $14.51 \pm 2.72$ & $14.4 \pm 2.90$ & 0.657 & $14.9 \pm 3.00$ & $14.06 \pm 2.59$ & 0.260 & 15.26 \\
\hline TP, g/L & $63.93 \pm 7.68$ & $63.78 \pm 10.34$ & 0.540 & $64.76 \pm 8.76$ & $63.05 \pm 9.56$ & 0.410 & 61.72 \\
\hline $\mathrm{PA}, \mathrm{mg} / \mathrm{dL}$ & $15.96 \pm 6.67$ & $17.71 \pm 6.29$ & 0.231 & $14.18 \pm 6.55$ & $18.88 \pm 5.74$ & $0.005^{* *}$ & 16.94 \\
\hline ALB, $g / L$ & $35.75 \pm 6.12$ & $36.84 \pm 6.93$ & 0.341 & $37.03 \pm 6.36$ & $35.75 \pm 6.74$ & 0.470 & 34.45 \\
\hline ALP, U/L & $106.79 \pm 81.75$ & $99.61 \pm 43.41$ & 0.583 & $104.84 \pm 59.66$ & $100.79 \pm 66.18$ & 0.793 & 102.42 \\
\hline $\mathrm{ALT}, \mathrm{U} / \mathrm{L}$ & $140.94 \pm 407.11$ & $57.24 \pm 61.02$ & 0.151 & $91.74 \pm 203.78$ & $98.2 \pm 333.43$ & 0.210 & 119.07 : \\
\hline AST, U/L & $94 \pm 364.89$ & $60.56 \pm 101.49$ & 0.249 & $59.69 \pm 101.85$ & $91.36 \pm 346.71$ & 0.502 & $43.86:$ \\
\hline GGT, U/L & $128.38 \pm 212.94$ & $94.02 \pm 122.55$ & 0.766 & $139.77 \pm 181.81$ & $83.2 \pm 154.87$ & 0.057 & $127.2 \pm$ \\
\hline $\mathrm{CHE}, 1000 \mathrm{U} / \mathrm{L}$ & $6.02 \pm 1.65$ & $5.96 \pm 1.71$ & 0.666 & $5.70 \pm 1.54$ & $6.22 \pm 1.74$ & 0.247 & $5.48:$ \\
\hline
\end{tabular}




\begin{tabular}{|c|c|c|c|c|c|c|c|}
\hline TBA, $\mu \mathrm{mol} / \mathrm{L}$ & $4.76 \pm 4.87$ & $4.16 \pm 5.86$ & 0.312 & $5.84 \pm 7.11$ & $3.36 \pm 2.95$ & 0.170 & $4.19=$ \\
\hline $\mathrm{DBIL}, \mu \mathrm{mol} / \mathrm{L}$ & $8.93 \pm 9.78$ & $5.77 \pm 2.84$ & 0.493 & $9.7 \pm 10.4$ & $5.66 \pm 3.36$ & 0.122 & $8.72:$ \\
\hline TBIL, $\mu \mathrm{mol} / \mathrm{L}$ & $17.95 \pm 14.17$ & $16.75 \pm 8.70$ & 0.568 & $20.2 \pm 14.67$ & $14.81 \pm 6.88$ & 0.103 & 18.37: \\
\hline CK, U/L & $186.1 \pm 220.13$ & $382 \pm 1184.96$ & 0.865 & $217.87 \pm 314.36$ & $\begin{array}{c}360.08 \pm \\
1186.69\end{array}$ & 0.940 & $287.88=$ \\
\hline CKMB, U/L & $17.95 \pm 9.85$ & $22.39 \pm 22.55$ & 0.343 & $18.16 \pm 8.53$ & $21.32 \pm 21.17$ & 0.930 & 18.23 \\
\hline Urea, mmol/L & $7.56 \pm 3.99$ & $7.94 \pm 8.41$ & 0.127 & $7.84 \pm 6.95$ & $7.7 \pm 6.56$ & 0.640 & $8.29=$ \\
\hline Uric, mmol/L & $273.5 \pm 159.03$ & $352.75 \pm 214.01$ & 0.280 & $332.93 \pm 185.1$ & $277.47 \pm 184.43$ & 0.213 & $314.57:$ \\
\hline Crea mmol/L & $91.22 \pm 43.83$ & $111.17 \pm 82.2$ & 0.789 & $110.42 \pm 66.54$ & $88.13 \pm 55.74$ & 0.083 & $99.33=$ \\
\hline CYSC, mg/L & $1.30 \pm 0.53$ & $1.42 \pm 0.95$ & 0.990 & $1.38 \pm 0.79$ & $1.34 \pm 0.73$ & 0.654 & $1.54:$ \\
\hline $\mathrm{Clq}, \mathrm{mmol} / \mathrm{L}$ & $182.2 \pm 35.56$ & $200.05 \pm 38.89$ & 0.172 & $177.55 \pm 36.91$ & $198.33 \pm 35.83$ & 0.074 & 181.63 \\
\hline $\mathrm{Na}, \mathrm{mmol} / \mathrm{L}$ & $138.48 \pm 4.85$ & $141.52 \pm 6.29$ & $0.037 *$ & $140.08 \pm 7.12$ & $140.15 \pm 4.48$ & 0.731 & 139.35 \\
\hline $\mathrm{K}, \mathrm{mmol} / \mathrm{L}$ & $3.63 \pm 0.87$ & $4.27 \pm 0.66$ & $0.015^{*}$ & $3.92 \pm 0.62$ & $3.84 \pm 1.02$ & 0.724 & $4.18=$ \\
\hline $\mathrm{CL}, \mathrm{mmol} / \mathrm{L}$ & $103.36 \pm 5.92$ & $111.18 \pm 7.39$ & $0.000 * * *$ & $104.75 \pm 8.74$ & $108 \pm 5.91$ & 0.052 & 105.88 \\
\hline $\mathrm{HCO}_{3}, \mathrm{mmol} / \mathrm{L}$ & $24.15 \pm 2.41$ & $23.98 \pm 2.95$ & 0.767 & $24.37 \pm 2.47$ & $23.76 \pm 2.92$ & 0.236 & 23.71 \\
\hline $\mathrm{PH}$ value & $6.93 \pm 0.69$ & $6.49 \pm 1.08$ & 0.106 & $7.06 \pm 0.6$ & $6.44 \pm 1.02$ & $0.021 *$ & $6.5 \pm$ \\
\hline $\mathrm{TC}, \mathrm{mmol} / \mathrm{L}$ & $4.19 \pm 0.83$ & $4.59 \pm 1.17$ & 0.255 & $4.62 \pm 1.32$ & $4.3 \pm 0.84$ & 0.589 & $4.92:$ \\
\hline $\mathrm{TG}, \mathrm{mmol} / \mathrm{L}$ & $1.34 \pm 0.56$ & $1.34 \pm 0.67$ & 0.834 & $1.24 \pm 0.47$ & $1.41 \pm 0.71$ & 0.677 & $1.5 \pm$ \\
\hline ApoAl, mmol/L & $1.14 \pm 0.18$ & $1.21 \pm 0.22$ & 0.469 & $1.18 \pm 0.17$ & $1.18 \pm 0.22$ & 0.938 & $1.14:$ \\
\hline ApoB, mmol/L & $0.85 \pm 0.22$ & $0.76 \pm 0.13$ & 0.674 & $0.75 \pm 0.22$ & $0.83 \pm 0.13$ & 0.277 & $0.85=$ \\
\hline LDL-C, mmol/L & $2.58 \pm 0.78$ & $2.87 \pm 1.06$ & 0.246 & $2.87 \pm 1.04$ & $2.68 \pm 0.92$ & 0.620 & $3.23=$ \\
\hline $\mathrm{HDL}-\mathrm{C}, \mathrm{mmol} / \mathrm{L}$ & $1.08 \pm 0.34$ & $1.22 \pm 0.31$ & 0.139 & $1.18 \pm 0.25$ & $1.16 \pm 0.37$ & 0.649 & $1.09=$ \\
\hline LDH, U/L & $451.88 \pm 779.15$ & $487.03 \pm 489.75$ & 0.138 & $505.85 \pm 538.16$ & $444.28 \pm 694.87$ & 0.171 & $410.67=$ \\
\hline INR & $1.14 \pm 0.22$ & $1.08 \pm 0.11$ & 0.446 & $1.13 \pm 0.21$ & $1.09 \pm 0.13$ & 0.172 & $1.09=$ \\
\hline PT, s & $13.11 \pm 2.41$ & $13.28 \pm 1.33$ & 0.149 & $13.51 \pm 2.27$ & $12.96 \pm 1.51$ & 0.119 & $12.9=$ \\
\hline PTA, \% & $85.87 \pm 17.42$ & $89.28 \pm 12.04$ & 0.615 & $85.62 \pm 16.02$ & $89.38 \pm 13.59$ & 0.198 & 88.54: \\
\hline APTT, s & $32.28 \pm 5.28$ & $35.79 \pm 5.86$ & $0.008^{* *}$ & $34.39 \pm 6.11$ & $34.04 \pm 5.69$ & 0.948 & 34.42 \\
\hline FPG, mmol/L & $7.61 \pm 2.84$ & $6.92 \pm 2.05$ & 0.355 & $7.2 \pm 1.92$ & $7.22 \pm 2.81$ & 0.281 & $6.38=$ \\
\hline
\end{tabular}




$\begin{array}{lccccccc}\text { CRP, } \mathrm{mg} / \mathrm{L} & 95.73 \pm 60.92 & 70.53 \pm 76.14 & 0.115 & 90.4 \pm 83.01 & 71.92 \pm 61.85 & 0.597 & 79.43: \\ \text { D-Dimer, } \mu \mathrm{g} / \mathrm{mL} & 2.02 \pm 3.57 & 4.10 \pm 5.73 & 0.187 & 3.25 \pm 4.94 & 3.09 \pm 5.02 & 0.234 & 1.81: \\ \mathrm{SAA}, \mu \mathrm{g} / \mathrm{mL} & 4.22 \pm 0.33 & 4.58 \pm 0.48 & 0.595 & 4.61 \pm 0.42 & 4.21 \pm 0.45 & 0.582 & 5.28:\end{array}$

Note,

BMI, Body Mass Index; HR, heart rate; WBC, white blood cells; RBC, red blood cells; HGB, hemoglobin; PLT, Platelet; BA, ba corpuscular hemoglobin; MCHC, mean corpuscular hemoglobin concentration; MCV, mean corpuscular Volume; MPV, mear distribution width; RDW, red blood cell volume distribution width; APTT, activated partial thromboplastin time; PT, prothror kinase; CKMB, creatine kinase isoenzyme MB; CRP, C-reactive protein; ALB, albumin; ALP, alkaline phosphatase; ALT, alani total bilirubin; $\mathrm{CHE}$, cholinesterase; GGT, gamma glutamyl transpeptidase; TBA, total bile acid; TP, total protein; PA, pre-al Creatinine; CYSC, Cystatin C; K, serum kalium; ApoA1, apolipoprotein A-1; ApoB, apolipoprotein B; FPG, fasting plasma glu triglycerides; LDL-C, low-density lipoprotein-cholesterol.

$* \mathrm{P}<0.05 ; * * \mathrm{P}<0.01 ; * * * \mathrm{P}<0.001$ 
Table 4 The correlations between SAA levels and clinical features in AAD patients

\begin{tabular}{|c|c|c|c|c|c|}
\hline $\begin{array}{l}\text { Characterizatio } \\
\text { n }\end{array}$ & Indices & $\bar{R}$ & P value & $\begin{array}{l}\text { Characterizatio } \\
\mathrm{n}\end{array}$ & Indices \\
\hline \multicolumn{4}{|l|}{$\overline{\text { Baseline Data }}$} & \multicolumn{2}{|l|}{ Liver function } \\
\hline & Age, $\mathrm{Yr}$ & -0.045 & 0.584 & & ALB, g/L \\
\hline & BMI & 0.126 & 0.123 & & $A L P, U / L$ \\
\hline & $\mathrm{HR}, \mathrm{bmp}$ & 0.333 & $<0.001^{* * *}$ & & $\mathrm{ALT}, \mathrm{U} / \mathrm{L}$ \\
\hline \multirow[t]{18}{*}{ Blood routine } & & & & & AST, U/L \\
\hline & WBC, $\times 10^{9} / \mathrm{L}$ & 0.614 & $<0.001^{* * *}$ & & DBIL, umol/L \\
\hline & $\mathrm{RBC}, \times 10^{12} / \mathrm{L}$ & -0.111 & 0.354 & & TBIL, umol/L \\
\hline & HGB, g/L & -0.395 & $<0.001^{* * *}$ & & CHE, 1000U/L \\
\hline & $\mathrm{PLT}, \times 10^{9} / \mathrm{L}$ & 0.091 & 0.285 & & GGT, U/L \\
\hline & $\mathrm{BA}, \times 10^{9} / \mathrm{L}$ & -0.101 & 0.385 & & TBA, umol/L \\
\hline & EO, $\times 10^{9} / \mathrm{L}$ & -0.243 & $0.035 *$ & & TP, g/L \\
\hline & $\mathrm{LY}, \times 10^{9} / \mathrm{L}$ & -0.168 & 0.147 & & $\mathrm{PA}, \mathrm{mg} / \mathrm{dL}$ \\
\hline & $\mathrm{MO}, \times 10^{9} / \mathrm{L}$ & 0.085 & 0.468 & & $\mathrm{LDH}, \mathrm{U} / \mathrm{L}$ \\
\hline & $\mathrm{NE}, \times 10^{9} / \mathrm{L}$ & 0.257 & $0.025^{*}$ & \multicolumn{2}{|c|}{ Renal function and serum electrolyte } \\
\hline & $\mathrm{MCH}, \mathrm{pg}$ & -0.065 & 0.574 & & $\mathrm{Na}, \mathrm{mmol} / \mathrm{L}$ \\
\hline & $\mathrm{MCHC}, \mathrm{g} / \mathrm{L}$ & -0.202 & 0.080 & & $\mathrm{Clq}, \mathrm{mmol} / \mathrm{L}$ \\
\hline & $M C V, f L$ & 0.015 & 0.898 & & $\mathrm{HCO}_{3}, \mathrm{mmol} / \mathrm{L}$ \\
\hline & MPV, fL & -0.098 & 0.405 & & $\mathrm{CL}, \mathrm{mmol} / \mathrm{L}$ \\
\hline & $\mathrm{HCT}, \mathrm{L} / \mathrm{L}$ & -0.198 & 0.148 & & Crea, $\mathrm{mmol} / \mathrm{L}$ \\
\hline & PCT, L/L & -0.011 & 0.930 & & CYSC, mg/L \\
\hline & PDW, 10GSD & 0.158 & 0.189 & & $\mathrm{PH}$ value \\
\hline & RDW, \% & 0.171 & 0.166 & & $\mathrm{~K}, \mathrm{mmol} / \mathrm{L}$ \\
\hline \multicolumn{4}{|c|}{ Blood coagulation function } & & Urea, $\mathrm{mmol} / \mathrm{L}$ \\
\hline & APTT, s & -0.241 & 0.051 & & Uric, mmol/L \\
\hline & PT, s & 0.03 & 0.811 & \multicolumn{2}{|c|}{ Serum lipid profile } \\
\hline & PTA, \% & -0.258 & $0.037 *$ & & ApoAl, mmol/L \\
\hline & INR & 0.251 & $0.042 *$ & & ApoB, $\mathrm{mmol} / \mathrm{L}$ \\
\hline & D-Dimer, ug/ml & 0.206 & 0.097 & & $\mathrm{FBG}, \mathrm{mmol} / \mathrm{L}$ \\
\hline \multicolumn{4}{|c|}{ Cardiovascular injury-related parameters } & & $\mathrm{HDL}-\mathrm{C}, \mathrm{mmol} / \mathrm{L}$ \\
\hline & CK, U/L & -0.039 & 0.756 & & $\mathrm{TC}, \mathrm{mmol} / \mathrm{L}$ \\
\hline & CKMB, U/L & 0.213 & 0.198 & & $\mathrm{TG}, \mathrm{mmol} / \mathrm{L}$ \\
\hline \multicolumn{4}{|c|}{ Inflammatory response } & & LDL-C, mmol/L \\
\hline & $\mathrm{CRP}, \mathrm{mg} / \mathrm{L}$ & 0.442 & $0.001 * * *$ & & \\
\hline
\end{tabular}

Note,

BMI, Body Mass Index; HR, heart rate; WBC, white blood cells; RBC, red blood cells; HGB, hemoglobin;

PLT, Platelet; BA, basophil; EO, eosinophil; LY, lymphocyte; MO, monocyte; NE, neutrophil; MCH, mean 
corpuscular hemoglobin; MCHC, mean corpuscular hemoglobin concentration; MCV, mean corpuscular Volume; MPV, mean platelet volume; HCT, hematocrit; PCT, platelet hematocrit; PDW, platelet distribution width; RDW, red blood cell volume distribution width; APTT, activated partial thromboplastin time; PT, prothrombin time; PTA, prothrombin activity, INR, international normalized ratio; CK, creatine kinase; CKMB, creatine kinase isoenzyme MB; CRP, C-reactive protein; ALB, albumin; ALP, alkaline phosphatase; ALT, alanine aminotransferase; AST, aspartate aminotransferase; DBIL, direct dilirubin; TBIL, total bilirubin; CHE, cholinesterase; GGT, gamma glutamyl transpeptidase; TBA, total bile acid; TP, total protein; PA, pre-albumin; LDH, lactate dehydrogenase; Na, serum sodium; CL, serum chlorine; Crea, Creatinine; CYSC, Cystatin C; K, serum kalium; ApoA1, apolipoprotein A-1; ApoB, apolipoprotein B; FPG, fasting plasma glucose; HDL-C, high-density lipoprotein-cholesterol; TC, total cholesterol; TG, triglycerides; LDL-C, low-density lipoproteincholesterol.

$* \mathrm{P}<0.05 ; * * \mathrm{P}<0.01 ; * * * \mathrm{P}<0.001$. 
Table 5 Diagnostic value of SAA and its combinations with CRP and D-Dimer for AAD patients

\begin{tabular}{|c|c|c|c|c|c|c|}
\hline & AUC & $95 \% \mathrm{Cl}$ & P-value & cut-off & $\begin{array}{c}\text { Sensitivity } \\
(\%)\end{array}$ & $\begin{array}{c}\text { Specificity } \\
(\%)\end{array}$ \\
\hline$\overline{A A D}$ & 0.942 & $0.902-0.981$ & $<0.001$ & 0.427 & 0.908 & 0.937 \\
\hline TAAD & 0.939 & $0.873-1.004$ & $<0.001$ & 0.427 & 0.919 & 0.937 \\
\hline TBAD & 0.937 & $0.883-0.990$ & $<0.001$ & 0.462 & 0.881 & 0.937 \\
\hline \multicolumn{7}{|l|}{ Combination } \\
\hline SAA+CRP & 0.977 & 0.955-0.999 & $<0.001$ & - & 0.944 & 0.938 \\
\hline $\begin{array}{l}\text { SAA+D- } \\
\text { Dimer }\end{array}$ & 0.9 & $0.838-0.963$ & $<0.001$ & - & 0.848 & 0.938 \\
\hline \multicolumn{7}{|l|}{ Death } \\
\hline $\begin{array}{l}\text { SAA (death } \\
\text { V.s. survival) }\end{array}$ & 0.732 & $0.612-0.853$ & 0.001 & 4.998 & 0.739 & 0.625 \\
\hline $\begin{array}{l}\text { CRP (death } \\
\text { v.s. survival) }\end{array}$ & 0.826 & $0.709-0.942$ & $<0.001$ & 82.55 & 0.789 & 0.771 \\
\hline $\begin{array}{l}\text { D-Dimer } \\
\text { (death v.s. } \\
\text { survival) }\end{array}$ & 0.715 & $0.562-0.869$ & 0.006 & 1.945 & 0.7 & 0.739 \\
\hline $\begin{array}{l}\text { NE\% (death } \\
\text { v.s. survival) }\end{array}$ & 0.678 & $0.549-0.808$ & 0.017 & 72.976 & 0.857 & 0.491 \\
\hline
\end{tabular}

Note, AAD-acute aortic dissection; TAAD-type A aortic dissection; TBAD-type B aortic dissection; SAA-serum amyloid A; CRP-C reactive protein; AUC-area under the curve; NE\%- neutrophil to lymphocyte ratio.

Table 6. Associations of parameters with the upper stratum of the SAA levels when compared with the lower stratum 


\begin{tabular}{|c|c|c|c|c|c|c|}
\hline & \multirow[t]{2}{*}{ SAA lower } & SAA upper & \multirow[t]{2}{*}{ P value } & & \multirow[t]{2}{*}{ SAA lower } & \multirow{2}{*}{$\mathrm{S}$} \\
\hline & & OR $(95 \% \mathrm{Cl})$ & & & & \\
\hline Age, Yr & 1 Reference & $0.98(0.92 ; 1.04)$ & 0.5343 & $\overline{A L B}, g / L$ & 1 Reference & 0.94 \\
\hline Hypertension, $\mathrm{n}$ & 1 Reference & $3.12(0.76 ; 12.84)$ & 0.1141 & ALP, U/L & 1 Reference & 1.01 \\
\hline Smoker, n & 1 Reference & $1.64(0.31 ; 8.54)$ & 0.5579 & ALT, U/L & 1 Reference & 1.01 \\
\hline D-Dimer, $\mu \mathrm{g} / \mathrm{mL}$ & 1 Reference & $1.72(0.79 ; 3.75)$ & 0.1752 & AST, U/L & 1 Reference & 1.00 \\
\hline CRP, mg/L & 1 Reference & $1.05(1.00 ; 1.09)$ & 0.0385 & GGT, U/L & 1 Reference & 1.00 \\
\hline CAD, $n$ & 1 Reference & $1.49(0.17 ; 13.14)$ & 0.7188 & $\mathrm{DBIL}, \mu \mathrm{mol} / \mathrm{L}$ & 1 Reference & 1.77 \\
\hline Tyре01, n & 1 Reference & $1.11(0.28 ; 4.50)$ & 0.8787 & TBIL, $\mu \mathrm{mol} / \mathrm{L}$ & 1 Reference & 1.02 \\
\hline WBC, $\times 10^{9} / \mathrm{L}$ & 1 Reference & $1.74(1.18 ; 2.56)$ & 0.0049 & $\mathrm{PA}, \mathrm{mg} / \mathrm{dL}$ & 1 Reference & 0.83 \\
\hline $\mathrm{RBC}, \times 10^{12} / \mathrm{L}$ & 1 Reference & $0.55(0.18 ; 1.66)$ & 0.2869 & $\mathrm{TP}, \mathrm{g} / \mathrm{L}$ & 1 Reference & 0.97 \\
\hline $\mathrm{HGB}, \mathrm{g} / \mathrm{L}$ & 1 Reference & $0.96(0.92 ; 1.00)$ & 0.0711 & TBA, $\mu \mathrm{mol} / \mathrm{L}$ & 1 Reference & 0.97 \\
\hline $\mathrm{PLT}, \times 10^{9} / \mathrm{L}$ & 1 Reference & $1.00(0.99 ; 1.01)$ & 0.9735 & $\mathrm{~K}, \mathrm{mmol} / \mathrm{L}$ & 1 Reference & 3. \\
\hline FPG, $\mathrm{mmol} / \mathrm{L}$ & 1 Reference & $1.17(0.80 ; 1.70)$ & 0.4191 & $\mathrm{CL}, \mathrm{mmol} / \mathrm{L}$ & 1 Reference & 0.98 \\
\hline $\mathrm{BA}, \times 10^{9} / \mathrm{L}$ & 1 Reference & $0.18(0.03 ; 1.20)$ & 0.0764 & $\mathrm{Na}, \mathrm{mmol} / \mathrm{L}$ & 1 Reference & 0.95 \\
\hline EO, $\times 10^{9} / \mathrm{L}$ & 1 Reference & $0.79(0.01 ; 114.81)$ & 0.9253 & $\mathrm{HDL}-\mathrm{C}, \mathrm{mmol} / \mathrm{L}$ & 1 Reference & 1.06 \\
\hline $\mathrm{LY}, \times 10^{9} / \mathrm{L}$ & 1 Reference & $0.85(0.77 ; 0.93)$ & 0.0004 & LDL-C, mmol/L & 1 Reference & 1.27 \\
\hline $\mathrm{MO}, \times 10^{9} / \mathrm{L}$ & 1 Reference & $1.02(0.94 ; 1.10)$ & 0.7075 & $\mathrm{TC}, \mathrm{mmol} / \mathrm{L}$ & 1 Reference & 1.40 \\
\hline $\mathrm{NE}, \times 10^{9} / \mathrm{L}$ & 1 Reference & $1.97(1.24 ; 3.11)$ & 0.0039 & $\mathrm{TG}, \mathrm{mmol} / \mathrm{L}$ & 1 Reference & 0.50 \\
\hline CKMB, u/L & 1 Reference & $1.01(0.89 ; 1.15)$ & 0.8363 & Urea, mmol/L & 1 Reference & 1.17 \\
\hline $\mathrm{MCH}, \mathrm{pg}$ & 1 Reference & $0.83(0.56 ; 1.21)$ & 0.3320 & Uric, mmol/L & 1 Reference & 1.01 \\
\hline $\mathrm{MCHC}, \mathrm{g} / \mathrm{L}$ & 1 Reference & $0.97(0.91 ; 1.04)$ & 0.4322 & Crea, mmol/L & 1 Reference & 1.09 \\
\hline $\mathrm{MCV}, \mathrm{fL}$ & 1 Reference & $0.95(0.84 ; 1.08)$ & 0.4593 & $\mathrm{Clq}, \mathrm{mmol} / \mathrm{L}$ & 1 Reference & 1.03 \\
\hline MPV, fL & 1 Reference & $0.77(0.47 ; 1.27)$ & 0.3056 & $\mathrm{HCO}_{3}, \mathrm{mmol} / \mathrm{L}$ & 1 Reference & 0.77 \\
\hline PCT, L/L & 1 Reference & $0.51(0.01 ; 3.93)$ & 0.9060 & $\mathrm{PH}$ value & 1 Reference & 0.08 \\
\hline PDW, 10GSD & 1 Reference & $1.03(0.80 ; 1.32)$ & 0.8293 & APTT, s & 1 Reference & 0.90 \\
\hline RDW, \% & 1 Reference & $2.43(0.99 ; 5.97)$ & 0.0535 & PT, s & 1 Reference & 1.08 \\
\hline $\mathrm{LDH}, \mathrm{U} / \mathrm{L}$ & 1 Reference & $1.01(1.00 ; 1.02)$ & 0.0634 & PTA, s & 1 Reference & 0.96 \\
\hline
\end{tabular}

Note,

HR, heart rate; WBC, white blood cells; RBC, red blood cells; HGB, hemoglobin; PLT, Platelet; BA, basophil; EO, eosinophil; LY, lymphocyte; MO, monocyte; NE, neutrophil; $\mathrm{MCH}$, mean corpuscular hemoglobin; MCHC, mean corpuscular hemoglobin concentration; MCV, mean corpuscular Volume; MPV, mean platelet volume; HCT, hematocrit; PCT, platelet hematocrit; PDW, platelet distribution width; RDW, red blood cell volume distribution width; APTT, activated partial thromboplastin time; PT, prothrombin time; PTA, prothrombin activity; CKMB, creatine kinase isoenzyme MB; CRP, C-reactive protein; ALB, albumin; ALP, alkaline phosphatase; ALT, alanine aminotransferase; AST, aspartate aminotransferase; DBIL, direct dilirubin; TBIL, total bilirubin; CHE, cholinesterase; GGT, gamma glutamyl transpeptidase; TBA, total bile acid; TP, total protein; PA, pre-albumin; LDH, lactate 
dehydrogenase; Na, serum sodium; CL, serum chlorine; Crea, Creatinine; CYSC, Cystatin C; K, serum kalium; ApoA1, apolipoprotein A-1; ApoB, apolipoprotein B; FPG, fasting plasma glucose; HDL-C, highdensity lipoprotein-cholesterol; TC, total cholesterol; TG, triglycerides; LDL-C, low-density lipoproteincholesterol.

Table 7 Univariate and multivariate logistic regression analyses for in-hospital mortality

\begin{tabular}{lcccc}
\hline & \multicolumn{2}{c}{ Model 1} & Model 2 \\
\hline & OR $(95 \% \mathrm{Cl})$ & P-value & OR $(95 \% \mathrm{Cl})$ & P-value \\
\hline Type of AAD & $0.47(0.16 ; 1.32)$ & 0.152 & $0.48(0.17 ; 1.36)$ & 0.167 \\
HR, bmp & $1.01(0.97 ; 1.05)$ & 0.584 & $1.01(0.97 ; 1.05)$ & 0.536 \\
Hypertension, $\%$ & $1.22(0.41 ; 3.64)$ & 0.724 & $1.13(0.37 ; 3.40)$ & 0.832 \\
Smoker, $\%$ & $0.63(0.20 ; 1.96)$ & 0.421 & $0.63(0.20 ; 2.00)$ & 0.432 \\
D-Dimer, $\mu \mathrm{g} / \mathrm{mL}$ & $1.19(0.98 ; 1.43)$ & 0.074 & $1.20(0.99 ; 1.46)$ & 0.065 \\
$\mathrm{CRP}, \mathrm{mg} / \mathrm{L}$ & $1.02(1.01 ; 1.03)$ & 0.000 & $1.03(1.01 ; 1.04)$ & 0.000 \\
$\mathrm{WBC}, \times 10^{9} / \mathrm{L}$ & $1.17(1.03 ; 1.32)$ & 0.013 & $1.17(1.03 ; 1.33)$ & 0.015 \\
$\mathrm{EO}, \times 10^{9} / \mathrm{L}$ & $0.68(0.40 ; 1.15)$ & 0.148 & $0.68(0.40 ; 1.16)$ & 0.161 \\
$\mathrm{LY}, \times 10^{9} / \mathrm{L}$ & $0.92(0.85 ; 0.99)$ & 0.030 & $0.92(0.85 ; 0.99)$ & 0.031 \\
$\mathrm{NE}, \times 10^{9} / \mathrm{L}$ & $1.05(1.00 ; 1.11)$ & 0.040 & $1.06(1.00 ; 1.11)$ & 0.041 \\
$\mathrm{HCO}, \mathrm{mmol} / \mathrm{L}$ & $0.69(0.53 ; 0.89)$ & 0.004 & $0.67(0.51 ; 0.88)$ & 0.004 \\
$\mathrm{LDH}, \mathrm{U} / \mathrm{L}$ & $1.00(0.99 ; 1.00)$ & 0.097 & $1.00(0.99 ; 1.00)$ & 0.151 \\
$\mathrm{FPG}, \mathrm{mmol} / \mathrm{L}$ & $1.17(0.95 ; 1.44)$ & 0.133 & $1.16(0.95 ; 1.43)$ & 0.149 \\
$\mathrm{SAA}, \mu \mathrm{g} / \mathrm{dL}$ & $1.22(1.05 ; 1.42)$ & 0.011 & $1.25(1.07 ; 1.47)$ & 0.005
\end{tabular}

Note,

$A A D$, acute aortic dissection; HR, heart rate; CRP, C reactive protein; WBC, white blood cell; EO, eosinophil; LY, lymphocyte; NE, neutrophil; LDH, lactate dehydrogenase; FPG, fasting plasma glucose; SAA, serum amyloid A, $\mathrm{Cl}$ : confidence interval, Model 1: no adjustments, Model 2: adjusted for age, gender, BMI

\section{Figures}




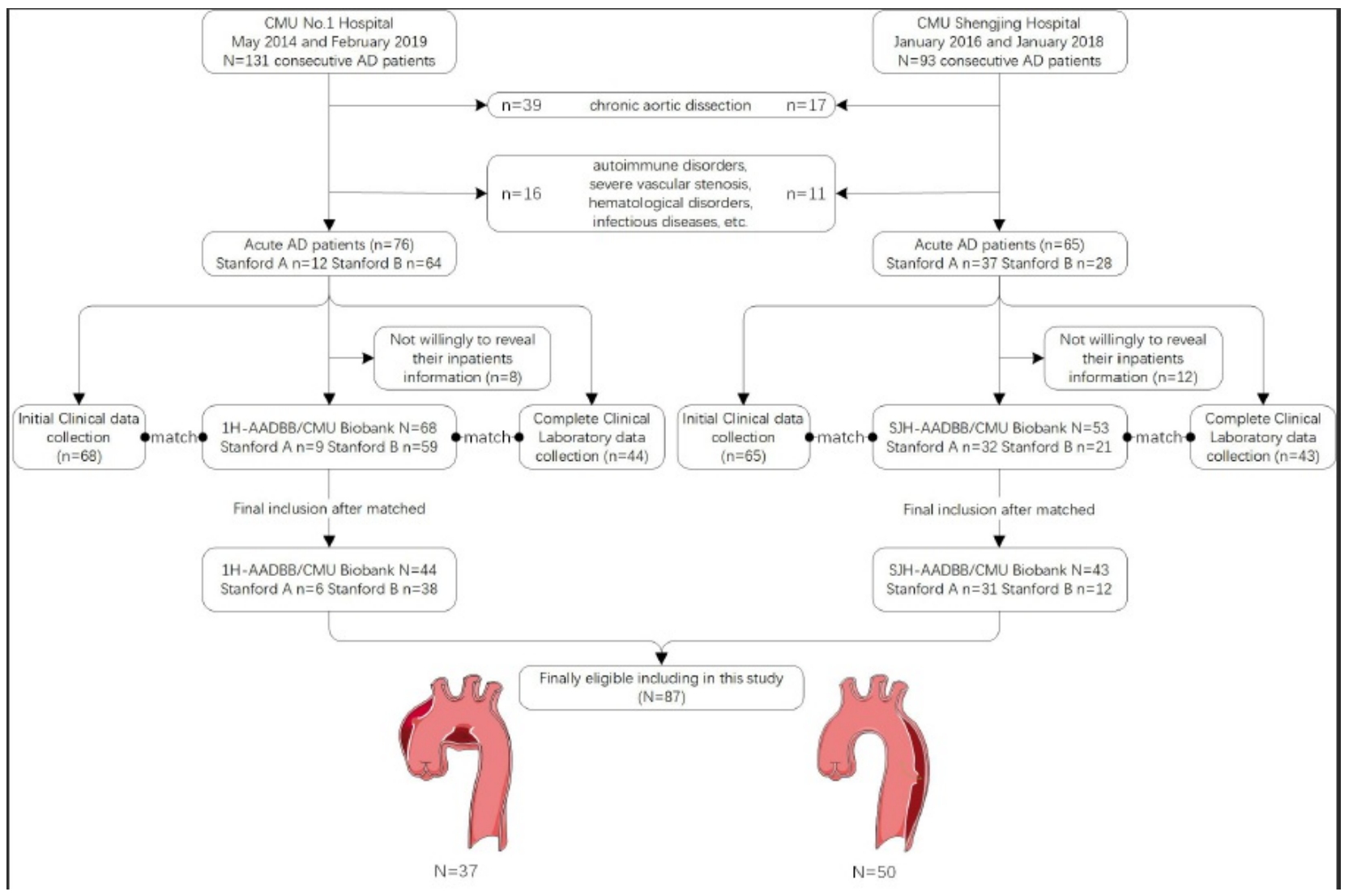

Figure 1

Flowchart of AAD patients selection and blood sample recovered from the First Hospital of CMU Aortic Dissection Blood sample Biobank (1H-ADBB/CMU) and Shengjing Hospital of CMU Aortic Dissection Blood sample Biobank (SJH-ADBB/CMU). 
A

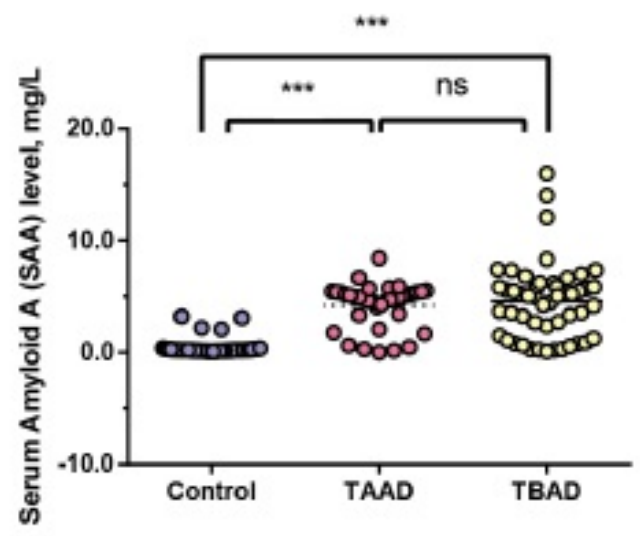

C

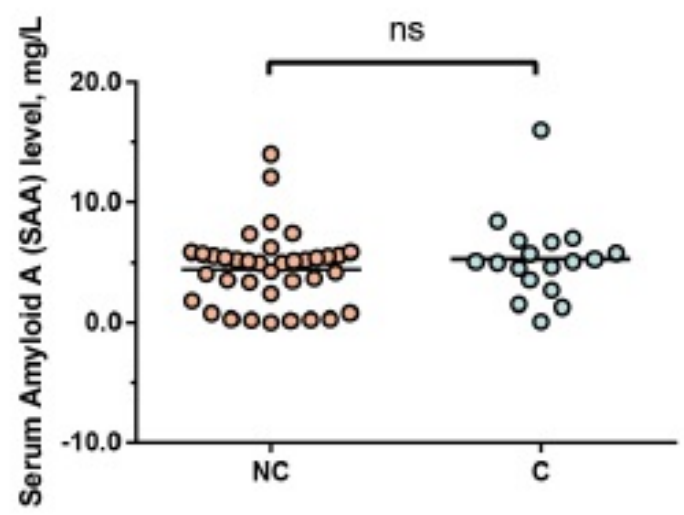

B

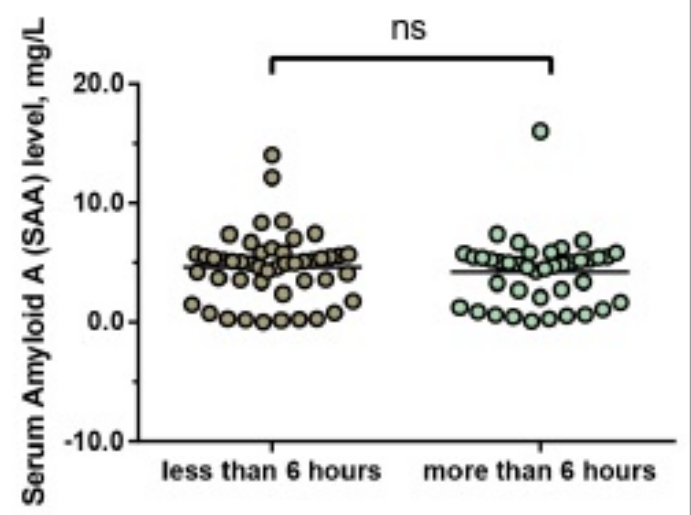

D

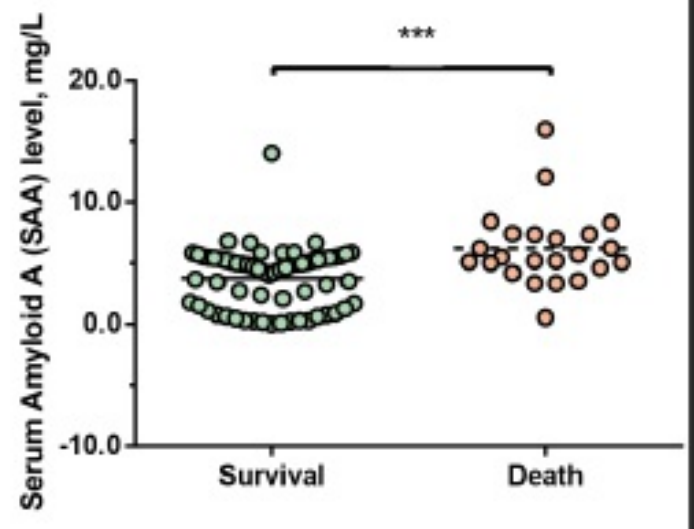

Figure 2

Analysis of SAA levels in different groups. (A) Comparison of SAA levels among controls, TAAD and TBAD. And sub-group analysis of SAA levels in AAD patients: (B)comparison of SAA levels between AAD with high-risk pain features that persisted less than six hours and more than six hours, and $(C)$ comparison of SAA levels between AAD with communicating and non-communicating false lumen (C and NC), and (D) comparison of SAA levels between death cases and survival cases. $* \mathrm{P}<0.05, * * \mathrm{P}<0.01, * * * \mathrm{P}<0.001$, ns, no significance. 
A

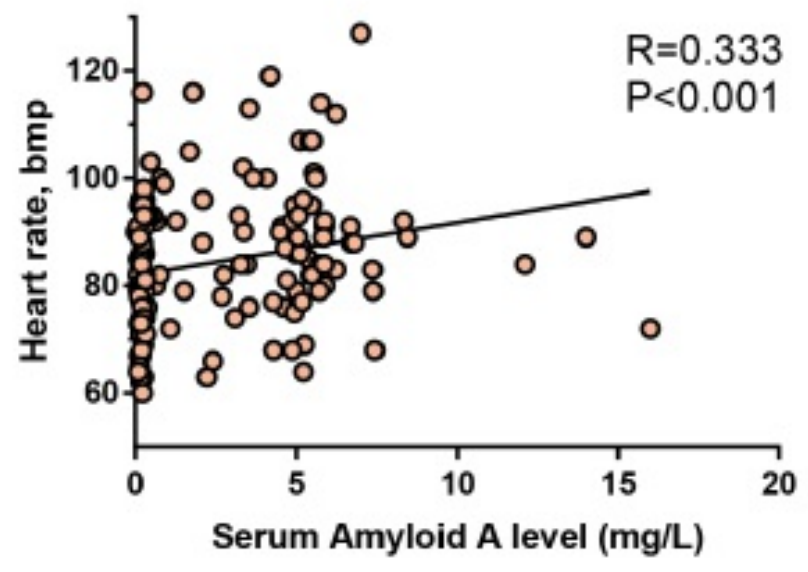

C

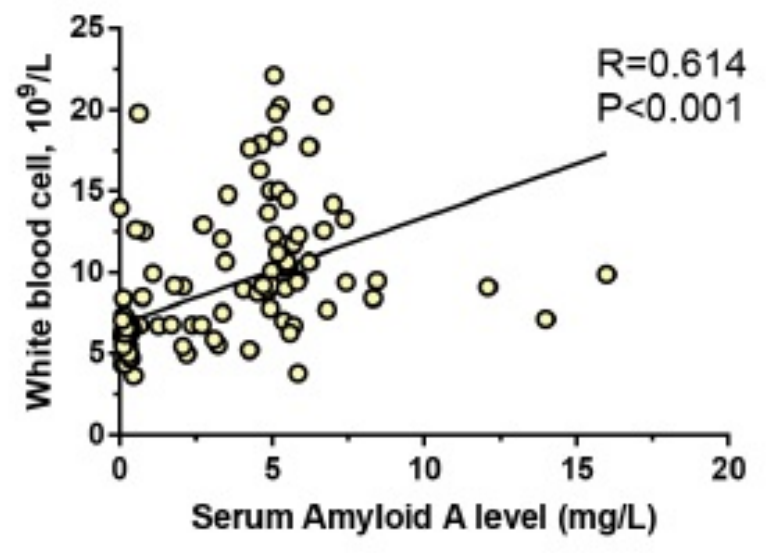

B

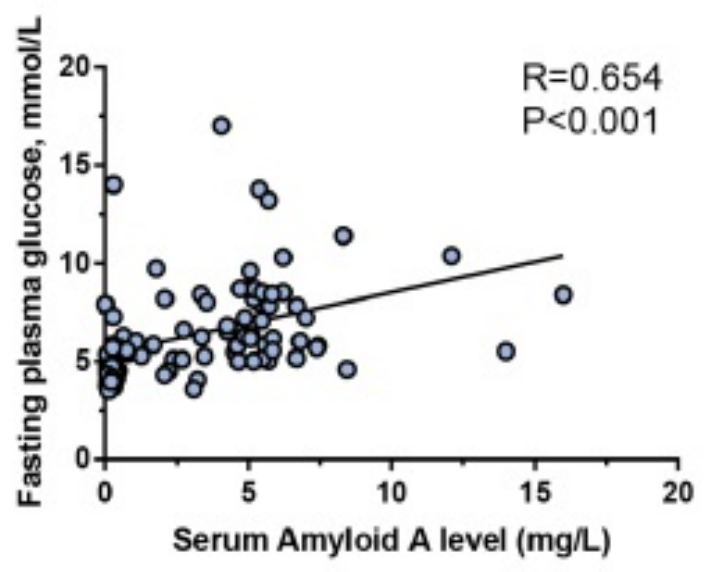

D

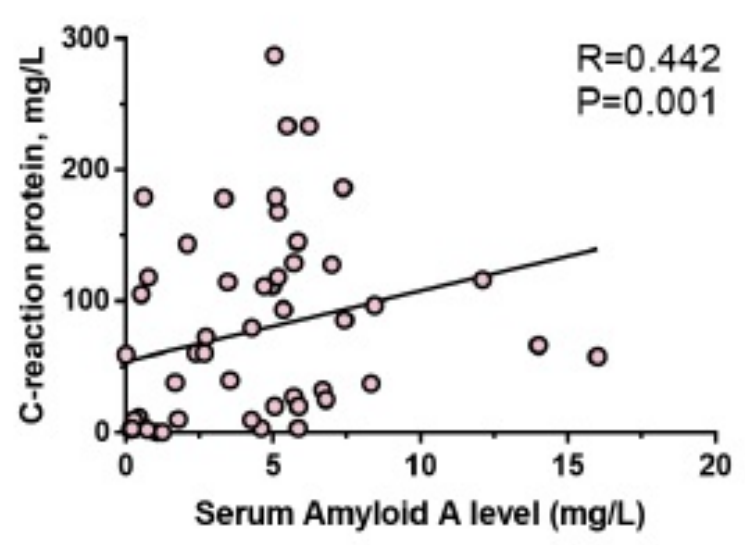

Figure 3

Correlations between SAA levels and clinical features in AAD patients. The horizontal axis represents SAA levels in $\mathrm{mg} / \mathrm{L}$ and the vertical axis represents (A)heart rate, bmp, (B)Fast plasma glucose, mmol/L, (C)white blood cell, 109/L, and (D)C-reactive protein, mg/L. 


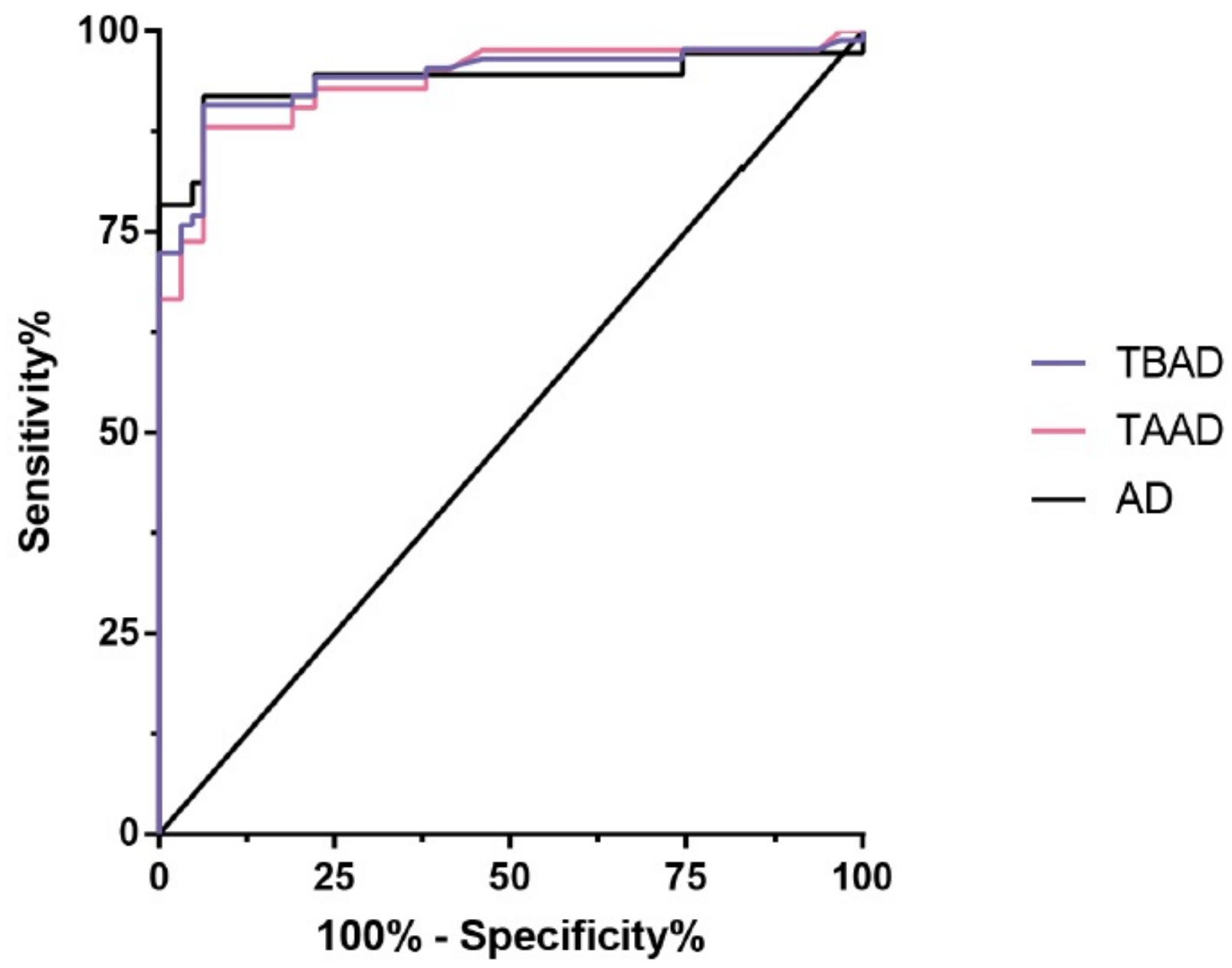

Figure 4

ROC analysis of SAA levels for the evaluation of TAAD, TBAD, and AAD. The vertical axis represents the sensitivity and the horizontal axis represents the 1-specificity 